\title{
ON THE ROLE OF LONG INCUBATION PERIODS IN THE DYNAMICS \\ OF ACQUIRED IMMUNODEFICIENCY SYNDROME (AIDS). \\ PART 2: MULTIPLE GROUP MODELS.
}

$\begin{array}{ll}\text { Wenzhang Huang } & \text { Carlos Castillo-Chavez } \\ \text { Claremont Grad. Sch. } & \text { Biometrics Unit } \\ \text { Claremont CA 91711 } & \begin{array}{l}\text { Cornell University } \\ \text { Ithaca, NY 14853 }\end{array}\end{array}$

\author{
Kenneth L. Cooke \\ Department of Math. \\ Pomona College \\ Simon A. Levin \\ Center for Env. Res. \\ Cornell University \\ Claremont, CA 91711
}

\begin{abstract}
In this paper, we restate previously obtained results on homogeneously-mixed single-group models for HIV (human immunodeficiency virus) with distributed waiting times in the infectious class, present some simulations that illustrate the effects of a changing mean sexual activity in the dynamics of HIV, and formulate a single group model for a heterogeneously mixed population with continuously-distributed sexual activity. This model forms the basis for our formulation of an N-group model with arbitrary social/sexual mixing. The local stability analysis of this $\mathrm{N}$-group model is discussed. A two-group example, under preferred mixing, that has multiple endemic equilibria is presented, as well as, an example for an N-group model, under proportionate mixing, possessing multiple endemic equilibria.
\end{abstract}

\section{Introduction}

The social/sexual mixing structure of a population or of a group of interacting populations plays a crucial role in the dynamics of disease transmission (see Kaplan et al (1989), Jacquez et al (1988,1989), Sattenspiel (1987), Sattenspiel and Simon (1988), May and Anderson (1989), Hyman and Stanley (1988,1989), Blythe and Castillo-Chavez (1989), and Castillo-Chavez and Blythe (1989)). These heterogeneities combined with the effects of the initial conditions and varying epidemiological and behavioral parameters can significantly affect the rates of disease spread within populations and among interacting populations.

An increased qualitative understanding of the role that social dynamics, variable infectivity (and other epidemiological parameters), asymptomatic carriers, age structure, socioeconomic structure, race, sexual preference, sexual behaviors (such as frequency of anal sex), sharing of needles, and intervention programs (such as the generalized use of AZT) play in the dynamics of HIV is necessary for the development, testing, and evaluation of control programs to slow down the AIDS epidemic at local and global scales.

\footnotetext{
${ }^{1}$ To whom all correspondence should be addressed.
} 
The increased quantitative evaluation of the effects of these factors in the dynamics of HIV within and between specific populations is of importance in the generation, through transmission models, of mid- and long-term predictions of the number of AIDS cases and the number of HIV infectives among these populations. In this paper, we introduce some fairly general models that incorporate some of the important features just discussed in a systematic way. The achievement of a qualitative understanding of the dynamics of this type of model through a combination of numerical simulations and mathematical analysis would represent a very important step toward their future validation.

This paper is organized into four sections. Section 2 introduces our basic single group model for a homogeneously mixed population. Our recent analytical results are stated, and the results of a few numerical simulations are presented to illustrate the effect of a varying mean sexual activity (a function of the effective sexually-active population size) in the dynamics of HIV. Section 3 introduces heterogeneous mixing through a continuous distribution of sexual activity: i.e., we divide our population by their degree (partners per unit time) of sexual activity. This approach is sometimes equated with the construction of a multiple group model. Although this is a valid interpretation, for practical purposes it may not be the most useful, as epidemiological data (such as AIDS incidence) are usually collected or aggregated by other criteria such as race, geographic location, socioeconomic background, drug use, sexual preference, etc. Of course, what is needed is a mixed approach where modelers, behaviorists, and epidemiologists work together to construct "strategic" models, which attempt to define the aggregation scheme (during the initial stages of research) and therefore have a direct input into experimental design (such as questionnaire development, or simply the gathering of data).

An aggregation scheme provides us with a collection of groups, each with its own distribution of sexual activity. A standard approach consists of assigning the mean sexual activity of its corresponding distribution to all members of such socially-defined groups. It is then assumed that group members mix at random within their own group, and a rule for intergroup mixing is then postulated (for a discussion on the limitations of this approach, see Sattenspiel 1987; Sattenspiel and Simon 1988). Of course, nobody prevents the modeler from constructing a more detailed multigroup model incorporating the distribution of sexual activity for each group. This last approach increases the level of detail and is important for theoretical reasons, as it allows us to determine the effects of some neglected features. However, it also has the disadvantage of increasing the number of parameters that will have to be estimated, and hence its value is diminished when we attempt to use it for predictive purposes. Since models can be used for very different purposes (see Hethcote and Yorke 1984), it is obvious that a balance has to be reached between the level of detail that one wishes to incorporate, the number of parameters that may be possible to estimate, and the effect of the level of aggregation that is considered appropriate for the question under consideration. 
Section 4 states our recent analytical results for multiple group models. A key result states that multiple group models can have multiple endemic equilibria even under the assumption of proportionate mixing. These results contradict the "generalized" thinking that epidemiological models of the SIR (susceptible, infected and removed) type have at most two equilibria, an infection-free and an endemic state. Two examples, for which two endemic equilibria are possible, are included in Section 5. A detailed technical exposition of these results will be published elsewhere (see Huang et al 1989).

\section{Single Population Models}

A sexually active homosexual population is subdivided into three groups: $\mathrm{S}$ (susceptible), I (HIV infectious), and A (AIDS infectious). We assume that A-individuals are sexually inactive and hence do not contribute to disease dynamics. Furthermore, we assume that sexually active individuals choose their partners at random. The demographic parameters are given by $\Lambda$, the recruitment rate into $S ; \mu$ gives the sexual activity removal rate; and $d$ specifies the AIDSinduced mortality rate. In addition, $\lambda$, which denotes the transmission rate per infectious partner, is assumed to be given by the product of two constant parameters: $i$, the average proportion of contacts with an infectious individual necessary for transmission, and $\phi$, the average number of contacts per sexual partner. $\mathrm{C}(\mathrm{T})$ denotes the mean number of sexual partners that an average individual has per unit time, given that the sexually active population is $\mathrm{T}=\mathrm{S}+\mathrm{I}$. It is reasonable to expect that in general $\mathrm{C}(\mathrm{T})$ increases linearly for small $T$ and saturates for large $T$. We further assume that the incidence rate $B(t)-$ the number of new cases per unit time-is proportional to $C(T)$, to $S$, and to the sexually active infected fraction:

$$
B(t)=\lambda C(T) S(t) \frac{I(t)}{T(t)}
$$

The proportionality constant is given by $\lambda$ (the transmission coefficient). Finally, we let $\mathrm{P}(\mathrm{s})$ denote the proportion of individuals infected at time $t$ and that, if alive, are still infectious at time $t+8$. Clearly $P(s)$ is nonnegative, nonincreasing, and $P(0)=1$. We assume that

$$
\int_{0}^{\infty} \mathrm{P}(\mathrm{s}) \mathrm{ds}<\infty
$$

and observe that $-\mathrm{P}^{\prime}(\mathrm{s})$ denotes the removal rate from group I into group $A, s$ time units after infection. The distributed-delay model for the sexual spread of HIV/AIDS is therefore given by the following system of integro-differential equations:

$$
\frac{d S(t)}{d t}=\Lambda-B(t)-\mu S(t),
$$




$$
\begin{aligned}
\mathrm{I}(\mathrm{t}) & =\mathrm{I}_{0}(\mathrm{t})+\int_{0}^{t} \mathrm{~B}(\mathrm{x}) \mathrm{e}^{-\mu(t-x)} \mathrm{P}(\mathrm{t}-\mathrm{x}) \mathrm{dx} \\
\mathrm{A}(\mathrm{t}) & =\mathrm{A}_{0}(\mathrm{t})+\mathrm{A}_{1} \mathrm{e}^{-\mathrm{dt} t}+\int_{0}^{t}\left\{\int_{0}^{\tau} B(x)\left[-\mathrm{P}^{\prime}(\tau-x) \mathrm{e}^{-\mathrm{d}(\mathrm{t}-\tau)}\right] \mathrm{dx}\right\} \mathrm{d} \tau
\end{aligned}
$$

where the functions (with compact support) $I_{0}(t), A_{0}(t)$, and the constant $A_{1}$, are introduced to take into account initial conditions.

This model generalizes the models developed by Anderson et al (1986), Anderson and May (1987), and Blythe and Anderson (1988a). In Blythe and Anderson (1988a) a submodel was studied numerically for various survivorship functions, and the local asymptotic stability analysis was completed for a specific family of survivorship functions. We (Castillo-Chavez et al $1989 \mathrm{a}, \mathrm{b}, \mathrm{c})$ have shown that this model has at most two attractors, which correspond to the infection-free state and the endemic state. In addition, we have completed a global stability analysis of the infection-free attractor for arbitrary survivorship functions. We have only been able to study the asymptotic local stability of the endemic attractor. We present here an outline of our results.

When $P(s)=e^{-\alpha s}$, the disease-free state $\left(\frac{\Lambda}{\mu}, 0\right)$ is a globally asymptotically stable equilibrium if and only if the reproductive number

$$
\mathrm{R} \equiv \lambda \mathrm{C}\left(\frac{\Lambda}{\mu}\right) \frac{1}{\mu+\alpha} \leq 1
$$

If $R>1$, there is a unique endemic state, which is a global attractor for all positive solutions.

When $P(s)$ is arbitrary, the infection-free state is a global attractor whenever the reproductive number

$$
\mathrm{R}=\lambda \mathrm{C}\left(\frac{\Lambda}{\mu}\right) \int_{0}^{\infty} \mathrm{e}^{-\mu \mathrm{s}} \mathrm{P}(\mathrm{s}) \mathrm{ds} \leq 1
$$

if $R>1$, then the limiting system

$$
\begin{aligned}
\frac{d S(t)}{d t} & =\Lambda-B(t)-\mu S(t), \\
I(t) & =\int_{-\infty}^{t} B(x) e^{-\mu(t-x)} P(t-x) d x,
\end{aligned}
$$

has a unique endemic state. This endemic state is locally asymptotically stable, provided that

$$
\frac{d M(T)}{d T} \leq 0, \text { where } M(T)=\frac{C(T)}{T} .
$$

The meaning of local asymptotic stability for this type of model can be found in Thieme and Castillo-Chavez (1989a).

In order to see the effects of $\mathrm{C}(\mathrm{T})$ on the dynamics of model (2)-(4), we simulate a special case, letting $P(8)$ denote a generalized gamma distribution. With this selection, our model reduces to the following system of ordinary differential equations: 


$$
\begin{aligned}
\frac{d S(t)}{d t} & =\Lambda-B(t)-\mu S(t), \\
\frac{d I_{1}(t)}{d t} & =B(t)-\left(a_{1}+\mu\right) I_{1}(t), \\
\frac{d I_{2}(t)}{d t} & =a_{1} I_{1}(t)-\left(a_{2}+\mu\right) I_{2}(t), \\
\frac{d I_{3}(t)}{d t} & =a_{2} I_{2}(t)-\left(a_{3}+\mu\right) I_{3}(t), \\
\frac{d I_{4}(t)}{d t} & =a_{3} I_{3}(t)-a_{4} I_{4}(t), \\
I_{4}(t) & =A(t), \\
\frac{d A_{T}(t)}{d t} & =a_{3} I(t),
\end{aligned}
$$

where $A_{T}(t)$ denotes the total number of AIDS cases at time $t$. The incidence in this case is given by the following expression:

$$
B(t)=C(T) S(t) \frac{\lambda_{1} I_{1}(t)+\lambda_{2} I_{2}(t)+\lambda_{3} I_{3}(t)}{T(t)}
$$

Since we intend to use approximately the same infectivity coefficients as those reported by Longini et al. (1989), we have taken only four compartments. Our simulations will use some of the current information available on the AIDS epidemic for the homosexual population living in San Francisco. These simulations do not attempt to reproduce the situation in San Francisco and are only used as an exploratory tool to increase our understanding of the mechanisms behind the AIDS epidemic. We note, for example, that the results presented by Hethcote at the 1989 SIAM summer meeting as understood by two of the above authors (hereafter referred as Hethcote 1989) show that in order to get a good fit to the San Francisco data (not available to the authors of this article), at least seven compartments are needed and a two-group population with biased mixing. In addition, Hethcote (1989) indicated that changes in behavior also need to be introduced through time-dependent parameters to explain the San Francisco data.

The simulations that follow are roughly based on the data presented by Hethcote (1989), the parameters estimated by Longini et al. (1989), and the data reported in the Los Angeles Times (December 6, 1988). Our main objective was to determine whether or not the simplest model (of the type that we have developed) was capable of fitting the reported data.

The simulation parameters were chosen as follows: $\phi_{1}=\phi_{2}=\phi_{3}=\phi=3 ; i_{1}=0.004$, $\mathrm{i}_{2}=0.000, \mathrm{i}_{3}=0.007 \mathrm{i}_{4}=0.0057$; hence $\lambda_{1}=0.012, \lambda_{2}=0.000, \lambda_{3}=0.021 \lambda_{4}=0.0171$. The removal rates (following Longini et al., 1989) selected were $a_{1}=5.0, a_{2}=0.23, a_{3}=0.19$, and 
$a_{4}=0.5$. We tried several two-parameter functional forms for $C(T)$ such as those described in Thieme and Castillo-Chavez (1989a,b) but were not able to get a good fit to the number of AIDS cases. To get a good fit it seemed necessary to increase the number of degrees of freedom required. In order to do this, an "ad hoc" procedure that maintained the required properties of $\mathrm{C}(\mathrm{T})$ was developed. We let $\mathrm{C}(\mathrm{T}) \equiv \phi \mathrm{C}(\mathrm{T})$ so that $\mathrm{C}(\mathrm{T})$ now denotes the number of unsafe contacts per unit time (one year). We took $T(0)=60,000$ and assumed that $T(t) \leq T(0)$, we further assumed that $\mathrm{C}(\mathrm{T})$ (a nondecreasing function of $\mathrm{T}$ ) was given by a step function that took on 15 values $r_{1} \leq r_{2} \leq \ldots \leq r_{15}$, with $C(T)=r_{1}$ whenever $T \leq 50,000$ and $C(60,000)=r_{15}$. The objective was to choose these 15 (possibly distinct) constants in such a way as to minimize the least square error fit to the number of AIDS cases. Initial simulations (which took up to 8 hours each in a VAX 750) reduced the viable range of $T$ to the $T$-interval $[59,000-60,000]$. We then repeated the same procedure in this $T$-interval but now using 40 "steps" $\left(r_{1} \leq r_{2} \leq \ldots \leq r_{40}\right)$. The initial data was chosen as $S(0)=59,600, I_{1}(0)=29, I_{2}(0)=$ $180, I_{3}(0)=161 . I_{4}(0)=30$ gives the reported number of AIDS cases in 1982 in San Francisco (see Table 1).

The best fit (continuous line) is illustrated in Figure 1. The mean square error is $\mathbf{2 0 2 4 . 6 2}$ and the standard deviation is 44.996. The fit is excellent at the beginning but not as good over the last two years. In Figure 2 a plot of the best $\mathrm{C}(\mathrm{T})$ (actually $\phi \mathrm{C}(\mathrm{T})$ ) is provided. We observe that great change in the mean number of unsafe contacts is needed around 1984 in order to obtain a good fit. The initial point given by $C(T)=900$ (not in Figure 2) is not indicative of the real situation but a consequence of the fitting procedure. $\mathrm{C}(\mathrm{T})$ drops immediately to 550 and ends at about 30 unsafe contacts per year. This models predicts approximately 9,000 HIV infecteds by the end of 1987 (Figure 3), which is quite in disagreement with those reported by Hethcote (1989) of 16,000 to 24,000 HIV infected individuals. Despite the good fit to the observed AIDS incidence, we did not get a good fit (with the model) to the AIDS prevalence (Figure 4). We observe that despite the tremendous flexibility of the model (which has a great number of parameters) the method (i.e., differential equations) is very constrictive. We need to fit not only a single curve such as the AIDS incidence (which here is done satisfactorily) but many others such as the AIDS prevalence, HIV incidence and prevalence, through curves generated by these systems of coupled differential equations with the same parameters. This apparent drawback is actually the biggest strength of the differential equation method in the process of validation of a model.

From these simulations we conclude that using Longini et al.'s (1989) data (and hence four compartments) is not possible to get a reasonable fit to AIDS incidence data using our simple model with homogeneous mixing and a saturating formula for $C(T)$. To fit the AIDS incidence data we have to use a computer intensive "ad hoc" method that properly constrains $C(T)$. Substantial behavioral changes are needed (average number of unsafe contacts per unit time) in order to fit these data. We further noticed that model predictions were somewhat 
sensitive to initial conditions. The difficulties encountered while trying to fit the AIDS incidence and prevalence as well as the HIV prevalence data with this simple model provided us with several important points: more detailed infectivity studies are needed, the assumption of homogeneous mixing is too restrictive-however, a simple form of mixing like preferred mixing with two or three groups may be sufficient to produce a better fit (unfortunately data on mixing is lacking)-changes in behavior (i.e., time-dependent parameters) may be critical to explain the data. These results seem to agree generally with those reported by Hethcote (1989). Finally, we note that data sets like the San Francisco data set on homosexual incidence and prevalence of HIV is critical to our fine tuning of AIDS models. This is of particular importance because of the observed sensitivity of these models to initial conditions.

2. Formulation of a model with arbitrary mixing

We consider a sexually active population that is stratified according to a continuous variable $s$ that measures the degree of sexual activity (number of sexual partners per unit time). Hence

$$
s+\int_{s}^{\Delta s} S(s, t) d s, \quad \int_{s}^{+\Delta s} I(s, t) d s, \text { and } \int_{s} s(s, t) d s
$$

denote the number of individuals in each of the epidemiological classifications - susceptible, infected, and "full-blown" AIDS - respectively, with sexual activity in the activity interval (s, $s+\Delta s)$. We again assume that A-individuals are sexually inactive; therefore, if $T(s, t)=S(s, t)$ $+\mathrm{I}(\mathrm{s}, \mathrm{t})$, then

$$
\int_{0}^{\infty} \mathrm{T}(\mathrm{s}, \mathrm{t}) \mathrm{d} \mathrm{s}=\hat{\mathrm{T}}(\mathrm{t})
$$

denotes the totally sexually active population at time t. Here, $C(s, W(T(., t))$ denotes the mean number of sexual partners per unit time that an individual with activity level s has, given that the sexually active population is $\hat{\mathrm{T}} . \mathrm{W}(\mathrm{T}(., \mathrm{t}))$ is a measure of total sexual availability, and here is chosen to be a functional of the size of the sexually active population. An example for which $\mathrm{W}$ depends only on total sexual availability, and hence is independent of $\mathrm{s}$, is given by

$$
W(t)=\int_{0}^{\infty} h(u) T(u, t) d u,
$$

where $\mathrm{h}(\mathrm{u})$ is an appropriate weighting function. The mixing function $\rho(s, r)$ (Blythe and Castillo-Chavez, 1989; Castillo-Chavez and Blythe, 1989) is such that

$$
\mathbf{r}+\int_{\mathbf{r}}^{\Delta \mathbf{r}} \rho(\mathrm{s}, \mathrm{u}) \mathrm{du}
$$


denotes the fraction of contacts of a person with activity level $s$ with persons with activity levels in $(r, r+\Delta r)$, and therefore satisfies the following constraints for all $8, r$, and $t$ :

$$
\begin{aligned}
\rho(\mathrm{s}, \mathrm{r}, \mathrm{t}) & \geq 0 \\
\int_{0}^{\infty} \rho(\mathrm{s}, \mathrm{r}, \mathrm{t}) \mathrm{d} \mathrm{r} & =1 \\
\rho(\mathrm{s}, \mathrm{r}, \mathrm{t}) \mathrm{C}(\mathrm{s}, \mathrm{W}(., \mathrm{t})) \mathrm{T}(\mathrm{s}, \mathrm{t}) & =\rho(\mathrm{r}, \mathrm{s}, \mathrm{t}) \mathrm{C}(\mathrm{r}, \mathrm{W}(., \mathrm{t})) \mathrm{T}(\mathrm{r}, \mathrm{t}) .
\end{aligned}
$$

Conditions (17) and (18) arise because $\rho(s, r, t)$ can be interpreted as a probability density function, while condition (19) expresses a conservation principle, i.e., that the total number of partnerships of s-people with r-people must equal the total number of partnerships of $r$-people with s-people.

To describe a dynamic model that incorporates a general mixing function, we have to introduce additional notation. We let $\Lambda(s)$ denote the recruitment rate into $S(s, t), \mu$ denotes the sexual removal rate, $d$ denotes the disease-induced mortality rate, and $\lambda(s, r)$ denotes the transmission coefficient between susceptible individuals with activity $s$ and infective individuals with activity $r$. Using this notation, we can now derive an expression for the number of new cases per unit time-the incidence $B(s, t)$. First, we observe that $I(r, t) \Delta r$ and $T(r, t) \Delta r$ give the total number of infective and sexually active individuals, respectively, with activity in the activity interval $(r, r+\Delta r)$. Hence,

$$
\frac{I(r, t)}{T(r, t)}
$$

denotes the infective fraction that has activity level in the activity interval $(r, r+\Delta r)$. Since $\rho(s, r, t) \Delta r$ denotes the proportion of partnerships that a typical individual with activity level $s$ has with persons with activity levels in the activity interval $(r, r+\Delta r)$ at time $t$, then $\mathrm{C}(s, \mathrm{~W}(\mathrm{~T}(., \mathrm{t})) \rho(s, r, t) \Delta \mathrm{r}$ denotes the average number of partnerships per person of activity $s$ with persons of activities in the interval $(r, r+\Delta r)$. Furthermore

$$
\mathrm{C}(\mathrm{s}, \mathrm{W}(\mathrm{T}(., \mathrm{t}))) \rho(\mathrm{s}, \mathrm{r}, \mathrm{t}) \Delta \mathrm{r} \frac{\mathrm{I}(\mathrm{r}, \mathrm{t})}{\mathrm{T}(\mathrm{r}, \mathrm{t})}
$$

denotes the fraction of the average number of partnerships per person of activity level $s$ that are with persons with activities in the interval $(r, r+\Delta r)$. The expression for the incidence therefore is given by

$$
\mathrm{B}(\mathrm{s}, \mathrm{t})=\mathrm{S}(\mathrm{s}, \mathrm{t}) \mathrm{C}\left(\mathrm{s}, \mathrm{W}(\mathrm{T}(., \mathrm{t})) \int_{0}^{\infty} \beta(\mathrm{s}, \mathrm{r}) \rho(\mathrm{s}, \mathrm{r}, \mathrm{t}) \frac{\mathrm{I}(\mathrm{r}, \mathrm{t})}{\mathrm{T}(\mathrm{r}, \mathrm{t})} \mathrm{dr} .\right.
$$

To model a population that mixes in proportion to their numbers and their sexual activity (i.e., proportionate mixing) we use the mixing function

$$
\rho(s, r, t)=\frac{C(r, W(T(., t))) T(r, t)}{\int_{0}^{\infty} C(u, W(T(., t))) T(u, t) d u},
$$


with its associated incidence rate

$$
B(s, t)=S(s, t) C\left(s, W(T(., t)) \int_{0}^{\infty} \beta(s, r) C(r, W(T(r, t)) I(r, t) d r .\right.
$$

More general mixing functions can be found in Blythe and Castillo-Chavez (1989) and CastilloChavez and Blythe (1989).

The simplest epidemiological model that incorporates these features is given by the following set of equations:

$$
\begin{aligned}
& \frac{\mathrm{dS}(\mathrm{s}, \mathrm{t})}{\mathrm{dt}}=\Lambda(\mathrm{s})-\mathrm{B}(\mathrm{s}, \mathrm{t})-\mu \mathrm{S}(\mathrm{s}, \mathrm{t}) \\
& \frac{\mathrm{dI}(\mathrm{s}, \mathrm{t})}{\mathrm{dt}}=\mathrm{B}(\mathrm{s}, \mathrm{t})-(\alpha(\mathrm{s})+\mu) \mathrm{I}(\mathrm{s}, \mathrm{t}), \\
& \frac{\mathrm{dA}(\mathrm{s}, \mathrm{t})}{\mathrm{dt}}=\alpha(\mathrm{s}) \mathrm{I}(\mathrm{s}, \mathrm{t})-\mathrm{d}(\mathrm{s}) \mathrm{A}(\mathrm{s}, \mathrm{t}),
\end{aligned}
$$

This model, as presented, is not an adequate model for the study of HIV dynamics, since it assumes a removal rate from the infective class $\alpha(\mathrm{s})$ independent of time since infection. This is not a crucial limitation, however, as the model can be modified easily to take into account not only time since infection but also different degrees of infectivity in the various infectious categories. The simplest way of doing so is by further subdividing the infectious class into several compartments with different removal rates (see Section 2); variable infectivity also is easily incorporated into the $\mathrm{B}(\mathrm{s}, \mathrm{t})$ term. A full model using partial differential equations can also be easily developed via the approach of Blythe and Anderson (1988b), Hyman and Stanley (1988, 1989), and Thieme and Castillo-Chavez (1989a,b). For extensions of these approaches to age-structured populations see Busenberg and Castillo-Chavez (1989a, b).

\section{Multiple group models}

In this section we describe an N-group model that is contained conceptually in models (23)-(25). A general type of mixing is discussed-biased or preferred mixing-that includes the familiar proportionate mixing. Analytical results for this general model are presented. To describe our N-group model, we proceed by introducing some new notation. Our $\mathrm{N}$ sexually active subpopulations are divided into three epidemiological classes: $S_{i}, I_{i}$, and $A_{i}$ for $i=1, \cdots, N . \Lambda_{i}$ denotes the constant recruitment rate of susceptibles into class $S_{i}, \mu$ denotes the sexual activity removal rate, $d_{i}$ denotes the disease-induced mortality in class $A_{i}$, and $\alpha_{i}$ denotes the $i^{\text {th }}$-removal rate of its corresponding infective class. Furthermore, $\lambda_{i j}$ denotes the transmission coefficient between group $i$ and group $j$ individuals. 
To describe the mixing, we let $p_{i j}(t)$ denote the fraction of new partnerships per unit time of individuals in group $j$ with individuals in group $i$. Then the $p_{i j}$ 's satisfy the following properties at all times:

$$
\begin{array}{cc}
\mathrm{p}_{\mathrm{ij}} \geq 0, & \mathrm{i}, \mathrm{j}=1, \cdots, N \\
\sum_{\mathrm{j}=1}^{N} \mathrm{p}_{\mathrm{ij}}=1, & \mathrm{i}=1, \cdots, N \\
\mathrm{C}_{\mathrm{i}}\left(\mathrm{W}\left(\mathrm{T}_{1}, \ldots \mathrm{T}_{\mathrm{N}}\right)\right) \mathrm{T}_{\mathrm{i}} \mathrm{p}_{\mathrm{ij}}=\mathrm{C}_{\mathrm{j}}\left(\mathrm{W}\left(\mathrm{T}_{1}, \ldots \mathrm{T}_{\mathrm{N}}\right)\right) \mathrm{T}_{\mathrm{j}} \mathrm{p}_{\mathrm{ji}}, & \mathrm{i}, \mathrm{j}=1, \cdots, N .
\end{array}
$$

Proportionate mixing is defined by

$$
p_{i j}^{(1)}=\frac{C_{j}\left(W\left(T_{1}, \ldots T_{N}\right)\right) T_{j}}{\sum_{k=1}^{N} C_{k}\left(W\left(T_{1}, \ldots T_{N}\right)\right) T_{k}}, \quad i, j=1, \cdots, N
$$

whereas preferred or biased mixing is given by

$$
\begin{aligned}
& p_{i j}^{(2)}=\left\{\begin{aligned}
f_{i}+\left(1-f_{i}\right) & \frac{C_{i}\left(W\left(T_{1}, \ldots T_{N}\right)\right)\left(1-f_{i}\right) T_{i}}{\hat{L}}, \quad i=j \\
\left(1-f_{i}\right) & \frac{C_{j}\left(W\left(T_{1}, \ldots T_{N}\right)\right)\left(1-f_{j}\right) T_{j}}{\hat{L}}, \quad i \neq j
\end{aligned}\right. \\
& \text { where } i, j=1, \cdots, N \text { and } \hat{L}=\sum_{k=1}^{N}\left(1-f_{k}\right) C_{k}\left(W\left(T_{1}, \ldots T_{N}\right)\right) \dot{T}_{k} \text {. }
\end{aligned}
$$

In the last definition $f_{i}$ denotes the fraction of group i's new partnerships per unit time that are reserved for within the $i^{\text {th }}$ subpopulation, while the remaining fraction, $1-f_{i}$, of group i's new partnerships per unit time is assumed to be distributed according to proportional mixing. Note that equation (23) includes proportional mixing (set $f_{i}=0$ for $i=1, \ldots, N$ ).

Following our discussion leading to equation (20), we conclude that the $\mathrm{i}^{\text {th }}$-incidence rate is given by

$$
B_{i}(t)=S_{i}(t) C_{i}\left(W\left(T_{1}, \ldots T_{N}\right)\right) \sum_{j=1}^{N} \lambda_{i j} p_{i j}(t) \frac{I_{j}(t)}{T_{j}(t)},
$$

where $T_{k}(t)=S_{k}(t)+I_{k}(t), k=1, \ldots, N$.

We set $\sigma_{i}=\frac{1 / \mu}{1 / \alpha_{i}}$ to rescale the dynamics of transmission and arrive at model (32)-(34):

$$
\begin{aligned}
& \frac{d S_{i}(t)}{d t}=\Lambda_{i}-B_{i}(t)-\mu S_{i}(t), \\
& \frac{d I_{i}(t)}{d t}=B_{i}(t)-\mu\left(\sigma_{i}+1\right) I_{i}(t), \\
& \frac{d A_{i}(t)}{d t}=\alpha_{i} I_{i}(t)-d_{i} A_{i}(t), \quad i=1,2, \cdots, N .
\end{aligned}
$$


- This model assumes constant removal rates from the infective classes into the AIDS classes. As noted before, this assumption can be relaxed easily by further subdivision of the infective classes, as illustrated in Section 2.

Models (32)-(34) have been analyzed in the case of preferred mixing when

$$
\mathrm{W}\left(\mathrm{T}_{1}, \ldots \mathrm{T}_{\mathrm{N}}\right)=\hat{\mathrm{T}}=\sum_{\mathrm{k}=1}^{\mathrm{N}} \mathrm{T}_{\mathrm{k}}
$$

To describe our results for this model, we define the probability $\kappa\left(i, T^{*}\right)$ by

$$
\kappa\left(\mathrm{i}, \mathrm{T}^{*}\right)=\frac{\mathrm{C}_{i}\left(\mathrm{~T}^{*}\right) \frac{\Lambda_{i}}{\mu}}{\sum_{k=1}^{\mathrm{N}} \mathrm{C}_{k}\left(\mathrm{~T}^{*}\right) \frac{\Lambda_{k}}{\mu}}, \text { where } \mathrm{T}^{*}=\sum_{k=1}^{\mathrm{N}} \frac{\Lambda_{k}}{\mu}, \quad \mathrm{i}=1,2, \cdots, \mathrm{N},
$$

and introduce the matrix $Q$ given by $\left[\frac{\mathrm{C}_{j}\left(\mathrm{~T}^{*}\right) \lambda_{i j} \kappa\left(\mathrm{i}, \mathrm{T}^{*}\right)}{\sigma_{i}+1}\right]_{\mathrm{N} \times \mathrm{N}}$, and the function $\mathrm{H}(\mu)=\mathrm{Q}-\mu \mathrm{E}$, where $\mathrm{E}$ is the $\mathrm{N} \times \mathrm{N}$ identity matrix.

The following local stability result for the general $\mathrm{N}$-group model was first reported in Castillo-Chavez et al. (1989c) for the case of proportionate mixing. It has since been extended to the case of preferred mixing (see Huang et al 1989). To describe it, we let $\mathrm{M}(\mathrm{H}(\mu))=$ $\sup \{\operatorname{Re} \rho: \operatorname{det}(\rho \mathrm{E}-\mathrm{H}(\mu))=0\}$.

There is a unique $\mu_{0}$ such that

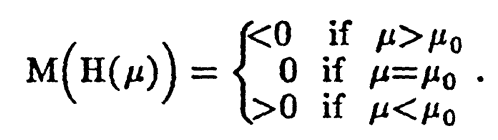

Furthermore the infection-free state $\bar{S}=\left(\frac{\Lambda_{1}}{\mu}, \cdots, \frac{\Lambda_{n}}{\mu}, 0, \cdots, 0\right)$ is locally asymptotically stable provided that $\mathrm{M}(\mathrm{H}(\mu))<0$.

Bifurcation results for the $\mathrm{N}$-group model were also reported in Castillo-Chavez et al (1989c) for the random mixing case. We have shown that the same assumptions as in CastilloChavez et al (1989c) - namely $\mathrm{C}_{\mathrm{i}}(\mathrm{T})=\mathrm{c}_{\mathrm{i}}$ (a constant) for $\mathrm{i}=1,2, \cdots, N, \mathrm{Q}$ is irreducible, and $\mu_{0}$ is such that $\mathrm{M}\left(\mathrm{H}\left(\mu_{0}\right)\right)=0$ - also hold for the preferred mixing case. To state our results, we introduce the expression

$$
\mathbf{h}\left(\mu_{0}\right)=\sum_{i=1}^{N} \bar{I}_{i} I_{i} \sum_{j=1}^{N}\left(c_{i} \lambda_{i j}-\mu_{0} \sigma_{j}\right) I_{j}
$$

where $I=\left(I_{1}, \cdots, I_{n}\right)$ and $T=\left(\Gamma_{1}, \cdots, \Gamma_{n}\right)$ are positive eigenvectors of $H\left(\mu_{0}\right)$ and $H^{T}\left(\mu_{0}\right)$ corresponding to the zero eigenvalue ( $\mathrm{T}$ denotes the transpose in this case). The existence of these positive eigenvectors (i.e., all entries are positive) is guaranteed by M-matrix theory. We (Huang et al 1989) have established the following bifurcation results:

If $\mathrm{h}\left(\mu_{0}\right) \neq 0$, then $\mu_{0}$ is a bifurcation point. More specifically, if $\mathrm{h}\left(\mu_{0}\right)>0\left(\mathrm{~h}\left(\mu_{0}\right)<0\right)$ 
then there is an $\epsilon>0$ and unique continuously differentiable functions $S$ and I mapping ( $\mu_{0}$ $\left.\epsilon, \mu_{0}\right] \rightarrow \mathrm{R}_{+}^{n}\left(\left[\mu_{0}, \mu_{0}+\epsilon\right) \rightarrow \mathrm{R}_{+}^{n}\right)$ such that $\left(\mathrm{S}\left(\mu_{0}\right), \mathrm{I}\left(\mu_{0}\right)\right)=\left(\frac{\Lambda_{1}}{\mu}, \cdots, \frac{\Lambda_{n}}{\mu}, 0, \cdots, 0\right)$, and $(\mathrm{S}(\mu), \mathrm{I}(\mu))$ is a positive endemic equilibrium of $(31, \mu)$. This endemic equilibrium is locally asymptotically stable for each $\mu$ in $\left(\mu_{0}-\epsilon, \mu_{0}\right)$ (unstable for each $\mu$ in $\left(\mu_{0}, \mu_{0}+\epsilon\right)$ ).

For each $\mu$ in $\left(0, \mu_{0}\right)$, the system $(31, \mu)$ has a positive endemic equilibrium; and if $\mathrm{h}\left(\mu_{0}\right)<$ 0 , there is an $\epsilon>0$, such that the system $(24, \mu)$ has at least two positive equilibria for each $\mu$ in $\left(\mu_{0}, \mu_{0}+\epsilon\right)$.

Our analytical results for models of the sexual spread of HIV/AIDS show that our single group models are robust, in the sense that only "simple" dynamics are possible. In addition (see Castillo-Chavez et al., 1989a,b), we have shown that the reproductive number is not significantly affected by the shape of the survivorship function, assuming that the survivorship function is biologically reasonable (but see Thieme and Castillo-Chavez 1989a,b). We have also illustrated the effects of a changing mean sexual activity, where the changes are due exclusively to a shrinking sexually active population. These changes allow us to obtain any kind of polynomial growth in the number of AIDS cases after the initial exponential growth phase exhibited by all models of this type. Furthermore, we see that the generalized thinking that S-I-R epidemic models do not have multiple equilibria is inaccurate, and hence the possibility for complex dynamics is certainly real. Our analytical results have been obtained under the assumption of preferred mixing; however, the model formulation is quite arbitrary, as the $p_{i j}(t)$ 's can be defined in a variety of ways as long as they satisfy the mixing constraints (25)-(27). Finally, we note that the above models have assumed that all infectious individuals are equally infectious. We have modified our single group model to include variable infectivity, and it appears that variable infectiousness does play a significant role in the qualitative dynamics of our single group model (see Thieme et al., 1989a,b). In addition, it can significantly affect quantitative values such as the initial rate of spread and the saturation level of cases. The numerical simulations found in Hyman and Stanley $(1988,1989)$ show that the transient dynamics for a similar model can be very sensitive to changes in the infectivity.

4. N-group model with endemic equilibria: two examples

The examples in this section illustrate the existence of multiple endemic equilibria for the $\mathrm{N}$-group epidemic model (equations $32-34$ ) of Section 3 . These examples point to the mechanism responsible for the generation of at least two endemic equilibria: asymmetry. Asymmetry arises through the nature of social/sexual interactions, that is, the mixing or asymmetric epidemiological parameters (here built in the transmission coefficient).

In order to introduce our examples we need some definitions. Let

$$
\theta_{i}=f_{i} \lambda_{i i} c_{i} \text {, }
$$




$$
M(T)=\Sigma r_{k} T_{k}, \quad r_{k}=c_{k}\left(1-f_{k}\right)
$$

and

$$
\left.\ell_{i j}=r_{i j} \lambda_{i j}=c_{i} c_{j} 1-f_{i}\right)\left(1-f_{j}\right) \lambda_{i j}
$$

With these definitions, equations (32)-(33) become

$$
\begin{aligned}
& \frac{d s_{i}}{d t}=\Lambda_{i}-\mu S_{i}-S_{i}\left(\frac{\theta_{i} I_{i}}{T_{i}}+\frac{1}{M(T)} \underset{j=1}{N} \ell_{i j} I_{j}\right) \\
& \frac{d I_{i}}{d t}=S_{i}\left(\frac{\theta_{i} I_{i}}{T_{i}}+\frac{1}{M(T)} \underset{j=1}{N} \ell_{i j} I_{j}\right)-\mu\left(s_{i}+1\right) I_{i}
\end{aligned}
$$

or equivalently in the form

$$
\begin{aligned}
& \frac{\mathrm{dS}}{\mathrm{dt}}=\mathrm{X}(\mu, \mathrm{S}, \mathrm{I}), \\
& \frac{\mathrm{dI}}{\mathrm{dt}}=\mathrm{Y}(\mu, \mathrm{S}, \mathrm{I}),
\end{aligned}
$$

where

$$
\begin{gathered}
S=\left(S_{1}, \cdots, S_{N}\right)^{T} \in R^{N}, \quad I=\left(I_{1}, \cdots, I_{N}\right)^{T} \in R^{N}, \\
\mu>0, \theta_{i} \geq 0, \ell_{i j} \geq 0, \Lambda_{i}>0, \sigma_{i}>0 \text { and } L=\left[\ell_{i j}\right]_{N \times N}
\end{gathered}
$$

is an irreducible matrix.

For a two-group example $(N=2)$, let $\mu_{0}=1, \sigma_{i}=1, \Lambda_{i}=4, \theta_{i}=1, \gamma_{i}=1, i=1,2$ and

$$
L=\left[\begin{array}{cc}
1-\epsilon & 0.1 \epsilon \\
1 & 0.9
\end{array}\right]
$$

where $\epsilon>0$. Lengthy computations then show that $h\left(\mu_{0}\right)<0$ (see Section 3) if $\epsilon$ is sufficiently small.

For our second example, we let $\Lambda_{i}=C_{i}=1, \sigma_{i}=\sigma>0, f_{i}=0, i=1, \cdots, N$, and $\lambda_{21}=\lambda_{31}=$ $\cdots=\lambda_{N 1} \lambda>0$ be fixed. We further let $\lambda_{11}>0, \lambda_{i j}=0, j \neq 1, i=1, \cdots, N$, and $\mu_{0}=\frac{\lambda_{11}}{N(\sigma+1)}$. For fixed $\sigma, \lambda>0$, we can then choose $\lambda_{11}>0$ small enough so that

$$
\mathrm{h}\left(\mu_{0}\right)=\lambda_{11}(1-\delta) 0,
$$

where

$$
\delta=\frac{\sigma}{N(\sigma+1)}+\frac{(N-1) \sigma \lambda}{N(\sigma+1) \lambda_{11}}
$$

For complete details see Huang et al. (1989). 


\section{Conclusion}

In this article we have restated some of our analytical results on single- and multiplegroup models. Of theoretical importance is the fact that multiple group models can possess multiple endemic equilibria. Two examples that illustrate this situation were presented in Section 5. We note, from these two examples, that what appears to be the critical factor in generating multiple endemic equilibria is asymmetry, either in mixing or in infectivity. There are, of course, several situations in which asymmetries of these types exist in the study of sexually transmitted diseases. Examples include the different infectivities for males and females found in gonorrhea research (see Hethcote and Yorke 1984), the (probable) asymmetric mixing between prostitutes and customers, etc.

The theoretical results generated by our models show that these asymmetries have an effect on the qualitative dynamics, and hence the importance of these asymetries depend on the parameters. Our results suggest strongly that experiments should be conducted to measure asymmetries that will answer important questions such as: How asymmetric is the mixing when one member of a pair is monogamous and the other is not? Is the probability of transmission from female to male and vice versa significantly different? How different is the probability of transmission between anal and vaginal intercourse? How asymmetric is the use of prophylactics such as condoms among sexual partners? etc.

We further note that without more detailed data on infectivity and mixing, and without further detailed epidemiological and behavioral studies (accessible to a variety of researchers) such as the San Francisco cohort studies, there is no hope that we can identify the relative effects of these key parameters and therefore increase our understanding of the AIDS epidemic. Predictions that go beyond those currently generated by statistical techniques will be difficult in the absence of these data.

\section{ACKNOWLEDGMENTS}

This research has been partially supported by NSF grant DMS- to K C, NSF grant DMS8906580 and Hatch project grant NYC 151-409, USDA to C C-C., and NSF grant DMS- to SAL. We thank them all for their generous support.

\section{REFERENCES}

Anderson, R. M., G. F. Medley, R. M. May and A. M. Johnson. (1986). A preliminary study of the transmission dynamics of the human immunodeficiency virus (HIV), the causative agent of AIDS. IMA J. of Mathematics Applied in Med. and Biol. 3, 229-263. 
Anderson , R. M. and R. M. May. (1987). Transmission dynamics of HIV infection. Nature $326,137-142$.

Blythe, S. P. and R. M. Anderson. (1988a). Distributed incubation and infectious periods in models of transmission dynamics of human immunodeficiency virus (HIV). IMA. J. of Mathmematics Applied in Med. and Biol. 5, 1-19.

Blythe, S. P. and R. M. Anderson. (1988b). Variable infectiousness in HIV transmission models. IMA J. of Mathematics Applied in Med. and Biol. 5, 181-200.

Blythe, S. P. and C. Castillo-Chavez. (1989). Like-with-like preference and sexual mixing models. Math. Biosci. (in press).

Busenberg, S. and C. Castillo-Chavez. (1989a). Interaction, pair formation and force of infection terms in sexaully transmitted diseases. (Submitted)

Busenberg, S. and C. Castillo-Chavez. (1989b). Risk and age-dependent mixing functions and force of infection terms in sexually transmitted diseases. (Submitted to IMA J. of Mathematics Applied in Med. and Biol.).

Castillo-Chavez, C. and S. P. Blythe. (1989). Mixing framework for social/sexual behavior. This volume.

Castillo-Chavez, C., K. Cooke, W. Huang and S. A. Levin. (1989a). On the role of long periods of infectiousness in the dynamics of acquired immunodeficiency syndrome (AIDS). In Mathematical Approaches to Problems in Resource Management and Epidemiology, C. Castillo-Chavez, S. A. Levin and C. Shoemaker, eds., Lecture Notes in Biomathematics, Springer-Verlag (in press).

Castillo-Chavez, C., K. Cooke, W. Huang and S. A. Levin. (1989b). On the role of long incubation periods in the dynamics of acquired immunodeficiency syndrome (AIDS), Part 1. Single population models, J. Math. Biol. (in press).

Castillo-Chavez, C., K. Cooke, W. Huang and S. A. Levin. (1989c). Results on the dynamics for models for the sexual transmission of the human immunodeficiency virus. Applied Mathematics Letters (in press).

Hethcote, H. W. (1989). A dynamic model of HIV transmission and AIDS in San Francisco. Lecture presented at the minisymposium on modeling the epidemiology of AIDS. 1989 SIAM Annual Meeting. July 17-21. San Diego, California.

Hethcote, H. W. and J. A. Yorke. (1984). Gonorrhea Transmission Dynamics and Control. Lecture Notes in Biomathematics 56. Spinger-Verlag, Heidelberg.

Huang, W., K. Cooke and C. Castillo-Chavez. (1989). Stability and bifurcation for a multiple group model for the dynamics of HIV/AIDS (manuscript).

Hyman, J. M. and E. A. Stanley. (1988). Using mathematical models to understand the AIDS epidemic. Math.Biosci. 90, 415-473.

Hyman, J. M. and E. A. Stanley (1989). The effect of social mixing patterns on the spread of AIDS. In Mathematical Approaches to Problems in Resource Management and Epidemiology. C. Castillo-Chavez, S. A. Levin and C. Shoemaker, eds., Lecture Notes in Biomathematics, Springer-Verlag (in press).

Jacquez, J. A., C. P. Simon and J. Koopman. (1989). Structured mixing: heterogeneous mixing by the definition of mixing groups. This volume.

Jacquez, J. A., C. P. Simon, J. Koopman, L. Sattenspiel and T. Perry. (1988). Modeling and analyzing HIV transmission: the effects of contact patterns. Math. Biosci. 92, 119-199.

Kaplan, E. H., P. C. Cramton and A. D. Paltiel. (1989). Nonrandom mixing models of HIV transmission. This volume.

Longini, I. M., Jr., W. S. Clark, M. Haber and C. R. Horsburgh, Jr. (1989). The stages of HIV infection: waiting times and infectious contact rates. This volume.

May, R. M. and R. M. Anderson. (1989). The transmission dynamics of human immunodeficiency virus (HIV). Phil. Trans. R. Soc. London $B$ 321, 565-607.

Sattenspiel, L. (1987). Population structure and the spread of disease. Human Biol. 59, 411438.

Sattenspiel, L. and C. P. Simon (1988). The spread and persistence of infectious diseases in structured populations. Math. Biosci. 90, 341-366.

Thieme, H. R. and C. Castillo-Chavez. (1989a). On the role of variable infectivity in the dynamics of the human immunodeficiency virus. This volume.

Thieme, H. R. and C. Castillo-Chavez. (1989b). On the possible efects of infection-agedependent infectivity in the dynamics of HIV/AIDS (manuscript). 
Table I.

$\begin{array}{rcrr}\text { Observation } & \begin{array}{c}\text { Time } \\ \text { (6 months) }\end{array} & \text { fitted } & \text { observed } \\ & & & \\ 1 & 0 & 30.590 & 30.628 \\ 2 & 1 & 33.095 & 33.592 \\ 3 & 2 & 49.861 & 56.316 \\ 4 & 3 & 102.916 & 128.440 \\ 5 & 4 & 190.765 & 157.092 \\ 6 & 5 & 285.253 & 229.216 \\ 7 & 6 & 371.723 & 329.004 \\ 8 & 7 & 446.573 & 397.176 \\ 9 & 8 & 508.933 & 434.720 \\ 10 & 9 & 560.226 & 545.376 \\ 11 & 10 & 602.764 & 685.672 \\ 12 & 11 & 638.707 & 686.660 \\ 13 & 12 & 668.015 & 680.732\end{array}$

Fitted and observed data from the simulation that generated the best fit (standard deviation 44.996). 


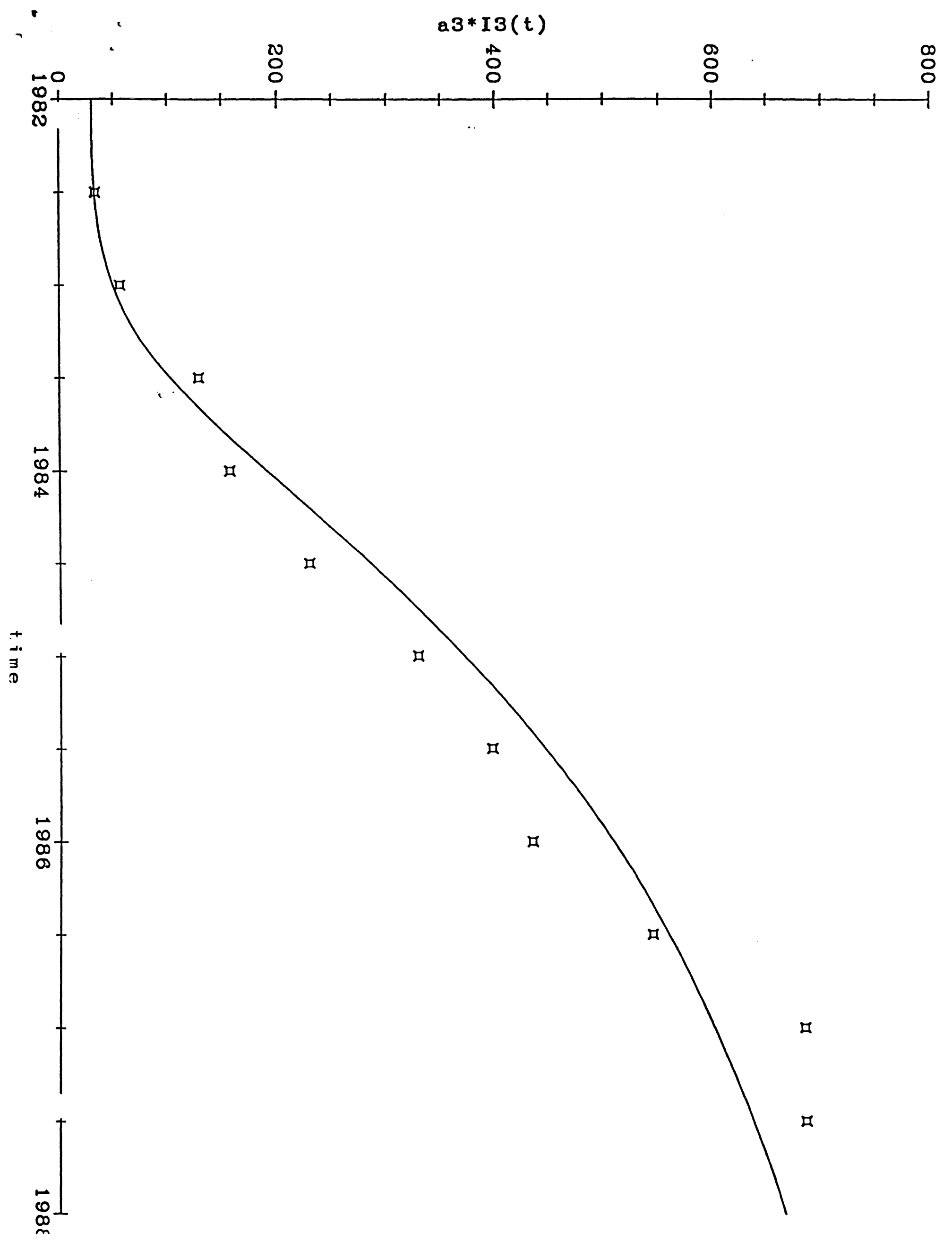


$C(T(t))$

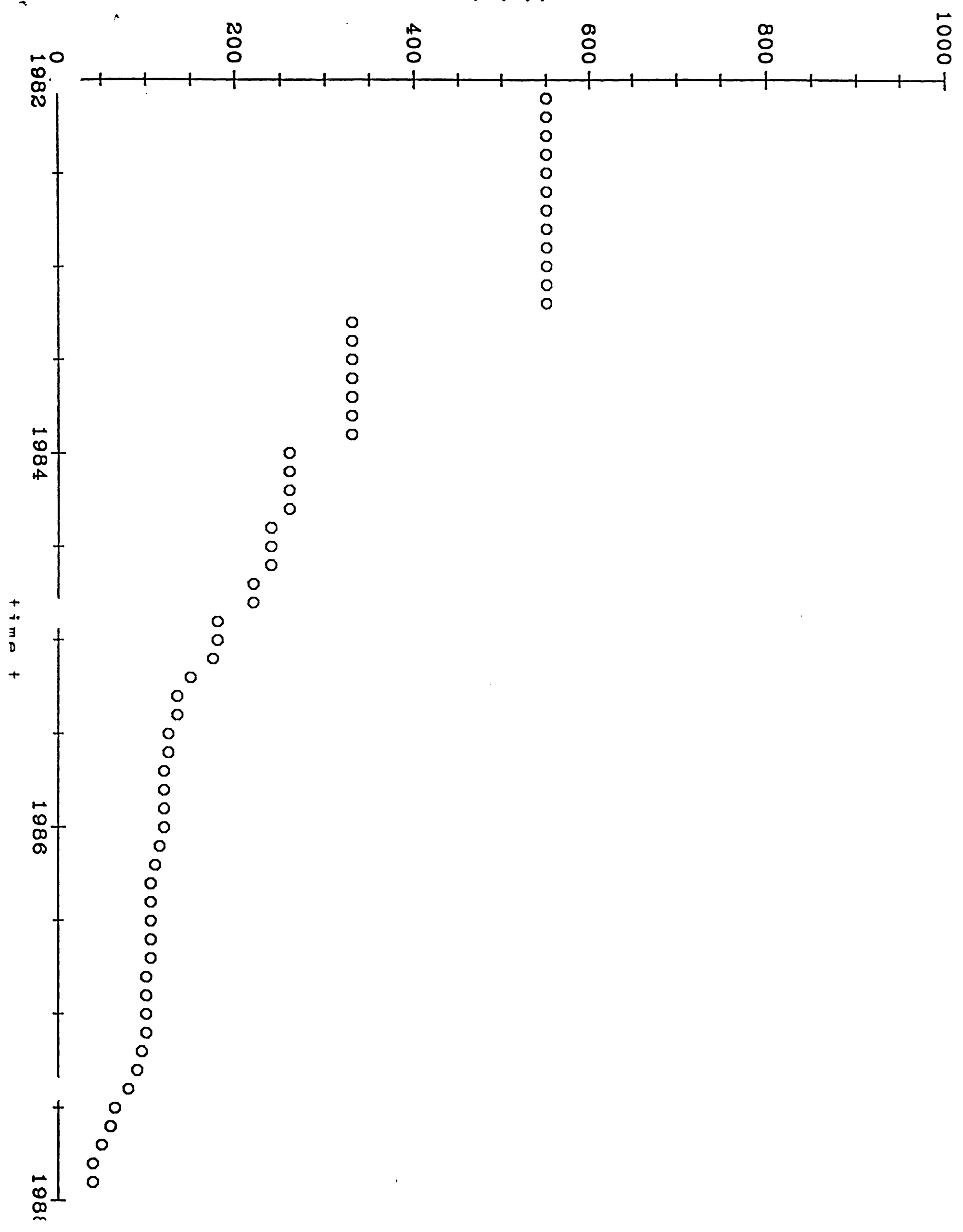







